\title{
Cytological Diagnosis of Duodenal Gangliocytic Paraganglioma on Ultrasound-Guided Fine Needle Aspirate: A Case Report with Review of the Literature
}

\author{
Arti Easwar*, Dion Middleton and Saverio Ligato \\ Department of Pathology and Laboratory Medicine, Hartford Hospital, USA
}

*Corresponding author: Arti Easwar, MD, Department of Pathology and Laboratory Medicine, Hartford Hospital, 80 Seymour Street, Hartford, CT 06102-5037, USA, Tel: 860-972-2488, Fax: 860-545-2204, E-mail: arti.easwar@hhchealth.org

\begin{abstract}
Duodenal gangliocytic paraganglioma (DGP) is a rare neoplasm usually found in the second portion of the duodenum and comprised of epithelioid neuroendocrine cells, Schwann cell-like spindle cells, and ganglion cells. We report a case of a 58-year-old male with a duodenal mass discovered incidentally during a staging PET scan for an oropharyngeal carcinoma. An ultrasound-guided fine needle aspirate (FNA) of the lesion revealed fascicles of spindle cells and sparse epithelioid cells with mild cytological atypia. Cell-block prepared from this specimen showed fragments of tightly packed clusters of spindle cells positive for S-100 and Sox 10. A diagnosis of a spindle cell neoplasm, favoring a schwannoma, was rendered. Subsequent surgical resection of this $2.8 \mathrm{~cm}$ submucosal mass showed predominantly spindled Schwann-like cells, interspersed epithelioid cells, rare ganglion cells, and an immunohistochemical profile that confirmed the diagnosis of DGP. On retrospective review of the prior aspirate, rare Neurofilament protein (NFP) positive ganglion cells were also identified and thus concluded to be consistent with DGP. Although two case reports of DGP, both with regional metastatic lymph nodal disease, have been previously described in the English language literature, this is the first case report describing the cytological features of a DGP without evidence of lymph node metastasis. In conclusion, in the evaluation of submucosal duodenal lesions by US-guided FNA, the possibility of a DGP should be included in the differential diagnosis when two or all three cell elements required for this diagnosis are identified and their phenotype is confirmed by immunohistochemical markers.
\end{abstract}

\section{Introduction}

Gangliocytic Paraganglioma (GP) is a rare neoplasm composed of a triphasic population of epithelioid cells, spindle cells, and ganglion-like cells. Although most commonly found in the second part of the duodenum, with a predilection for the periampullary region, rare cases with involvement of the jejunum, pylorus, esophagus, pancreas, appendix, and lung have also been reported in the literature [1]. DGP is generally considered a benign lesion, however, metastasis to regional lymph nodes or rarely to other organs, including one case with bone metastasis and two with liver metastasis, have also been reported in approximately $5-7 \%$ of cases [28]. Most cases are diagnosed on surgical resection, and to our knowledge, only two case reports describing the cytological features of DGP have been reported in the English language literature $[6,8]$. Interestingly, both cases were associated with metastasis to locoregional lymph nodes. Herein, we present the cytological features of a new case of DGP presenting as a solitary submucosal mass without evidence of lymph node metastasis. In addition, we will discuss the differential diagnosis with other submucosal duodenal lesions, and the limitations that sometimes may preclude its diagnosis on US-guided FNA.

\section{Case Description}

\section{Clinical history}

A 58-year-old male with a past medical history of oropharyngeal carcinoma, status post-chemoradiation therapy, underwent staging PET scan that revealed an incidental area of increased avidity in the second part of the duodenum close to the ampulla. He denied any gas- 


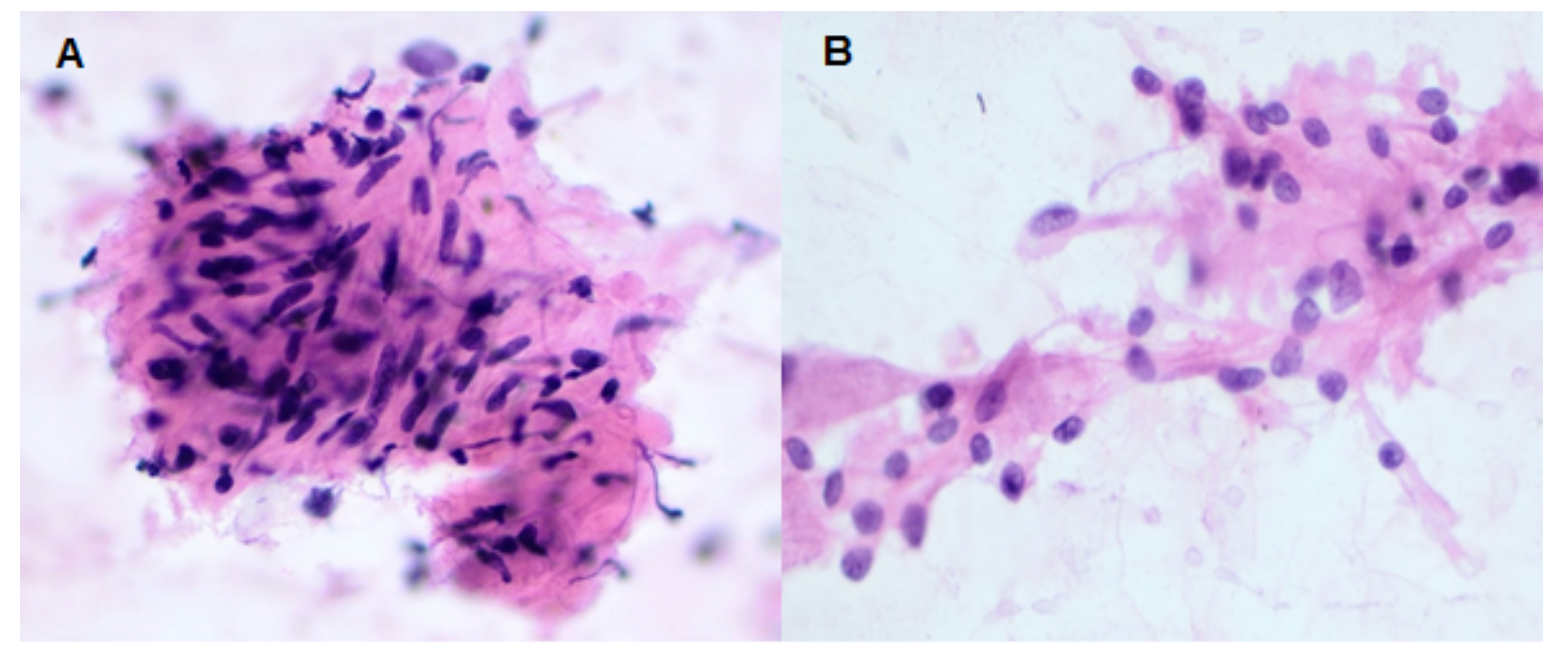

Figure 1: Hematoxylin and Eosin staining of the FNA specimen showing clusters of spindle cells (A) and medium to large epithelioid cells with ample eosinophilic cytoplasm and eccentric nuclei (B). (20X original magnification).

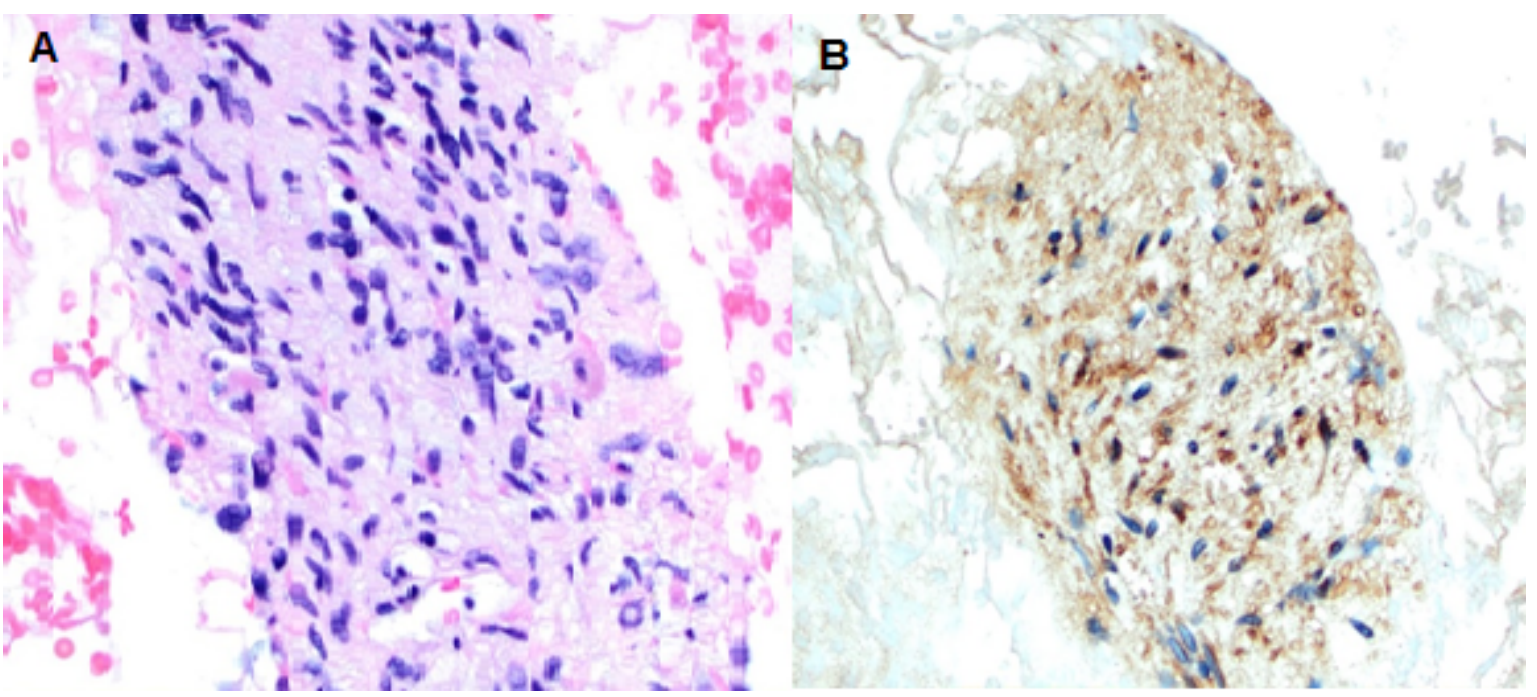

Figure 2: Hematoxylin and Eosin staining of the cell block showing predominantly spindle cells (A) (20X magnification). S100 expression highlights the spindle cells (B). (20X original magnification).

trointestinal symptoms that could be attributed to the duodenal mass. He underwent an endoscopic examination, which revealed a $1.8 \times 1.2 \mathrm{~cm}$ pedunculated subepithelial mass. An endoscopic biopsy was inconclusive and an US-guided FNA was performed.

\section{Cytopathological and histopathological findings}

The aspirate was predominantly composed of spindle cells arranged in fascicles (Figure 1a) and a few small groups of epithelioid cells with only mild cytological atypia (Figure 1b). Additionally, a cell-block prepared from the aspirate showed a few tissue fragments of tightly packed spindle cells (Figure 2a). The differential diagnosis included Schwannoma, Ganglioneuroma, Gastrointestinal Stromal Tumor (GIST) and Leiomyoma. Immunohistochemical studies performed on the cell block showed that the spindle cells were positive for S-100 (Figure 2b), and Sox 10, while negative for CD117, DOG1 and desmin; thus, excluding GIST and leiomyoma. A diagnosis of a spindle cell neoplasm favoring a schwannoma was rendered and the patient underwent partial duodenectomy, which revealed a smooth, tan, submucosal pedunculated lesion measuring $2.8 \mathrm{~cm}$ in greatest dimension, with a $2.0 \mathrm{~cm}$ stalk not involving the ampulla.

Histological examination showed a submucosal unencapsulated, but fairly well circumscribed, multilobulated nodule with three different cell types. The main cellular component consisted of spindle cells arranged in fascicles with tapered nuclei, inconspicuous nucleoli, and eosinophilic cytoplasm. The other two cell types featured nests of epithelioid cells supported by spindle-shaped sustentacular cells, and randomly distributed ganglion-like cells with abundant eosinophilic cytoplasm, large eccentric nuclei with vesicular chromatin, and prominent nucleoli (Figure 3). Mitoses were rare, and no necrosis was identified. Immunohistochemical studies showed strong positivity for S100 in the spindle cells, the epithelioid cells were positive for synaptophysin and chromogranin, while Neurofilament protein (NFP) was expressed only in the ganglion-like cells and their extensions (Figure 4). A diagnosis of DGP was ren- 


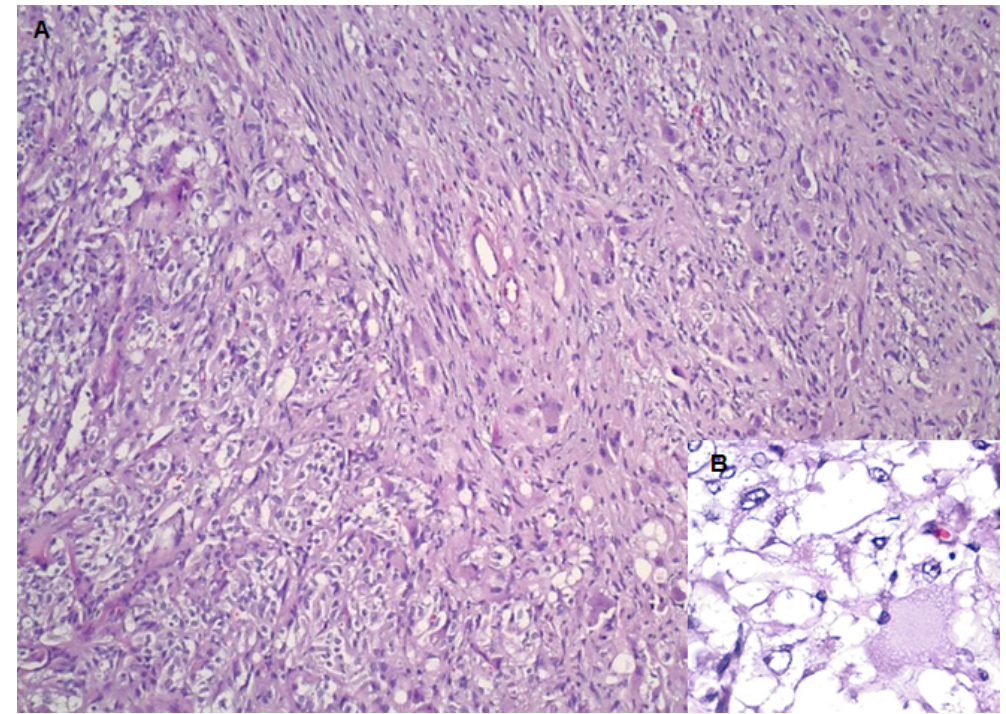

Figure 3: Hematoxylin and Eosin stain showing all three elements of gangliocytic paraganglioma - nests of epithelioid cells forming a Zellballen pattern, fascicles of spindled sustentacular cells, and scattered large ganglion cells (A - 20X original magnification). The insert (B) shows epithelioid cells and ganglion cells with eccentric nuclei and abundant eosinophilic cytoplasm. (40X original magnification).

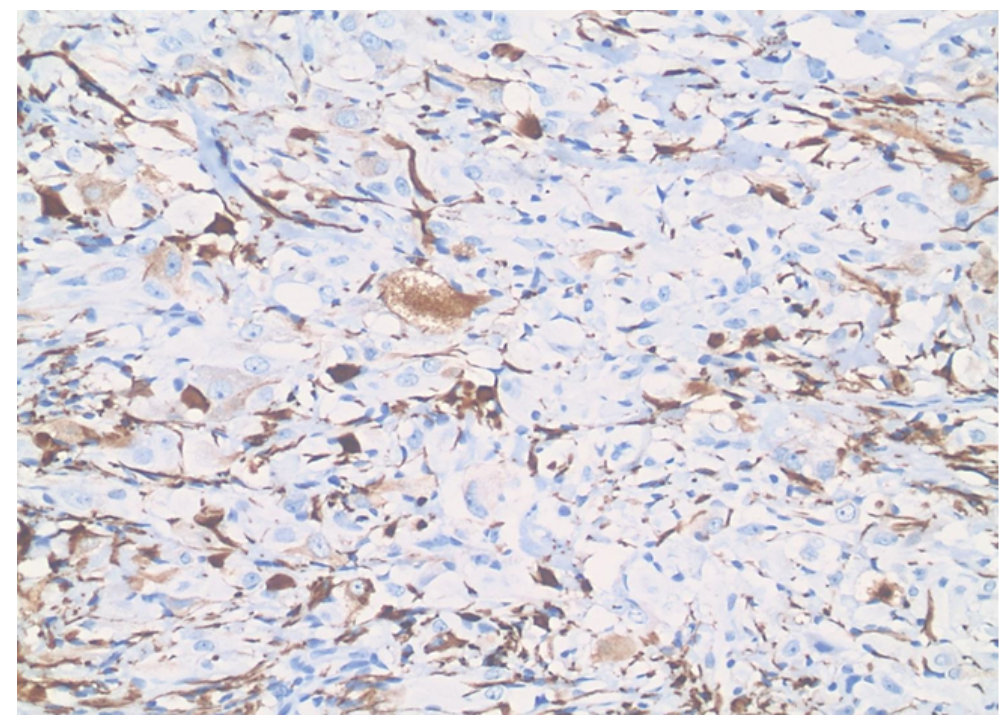

Figure 4: Immunohistochemical expression of Neurofilament protein in ganglion cells and axon-like extensions. (20X original magnification).

dered and the lesion was subsequently completely excised with clear margins. On follow-up, approximately 6 months after surgery, no clinical or radiological evidence of residual, recurrent, or metastatic disease was identified.

On retrospective review of the prior FNA, in addition to the spindle and epithelioid cells, a few polygonal cells cytologically resembling ganglion-like cells and immunohistochemically positive for NFP were identified; confirming the cytological diagnosis of DGP (Figure $5 \mathrm{a}$ and Figure $5 b$ ).

\section{Discussion}

DGP was first described by Dahl, et al. [9] as "duodenal ganglioneuroma". Subsequently, Kepes and Zacharias, due to its features similar to ganglioneuroma and paraganglioma, coined the term of Duodenal Gangliocytic Paraganglioma (DGP) [10]. Since then more than 200 cases have been reported so far in the literature [1]. Although the 2010 WHO classification consider DGP a benign neuroendocrine tumor [11], approximately $5-7 \%$ of cases have been reported to recur, have regional lymph node metastasis, demonstrate direct invasion of the nearby structures, or very rarely metastasize to other organs $[12,13]$. Therefore, recently, some authors have suggested to reclassify GP as a tumor of uncertain malignant potential $[7,13]$. Most of the patients with DGP are usually asymptomatic, and the lesion is found incidentally on routine examination. However, sometimes it may present with gastrointestinal bleeding, abdominal pain, anemia or jaundice. A male predominance, with the highest incidence in the $5^{\text {th }}-7^{\text {th }}$ decade, has also been described [1]. Some reports of functional 


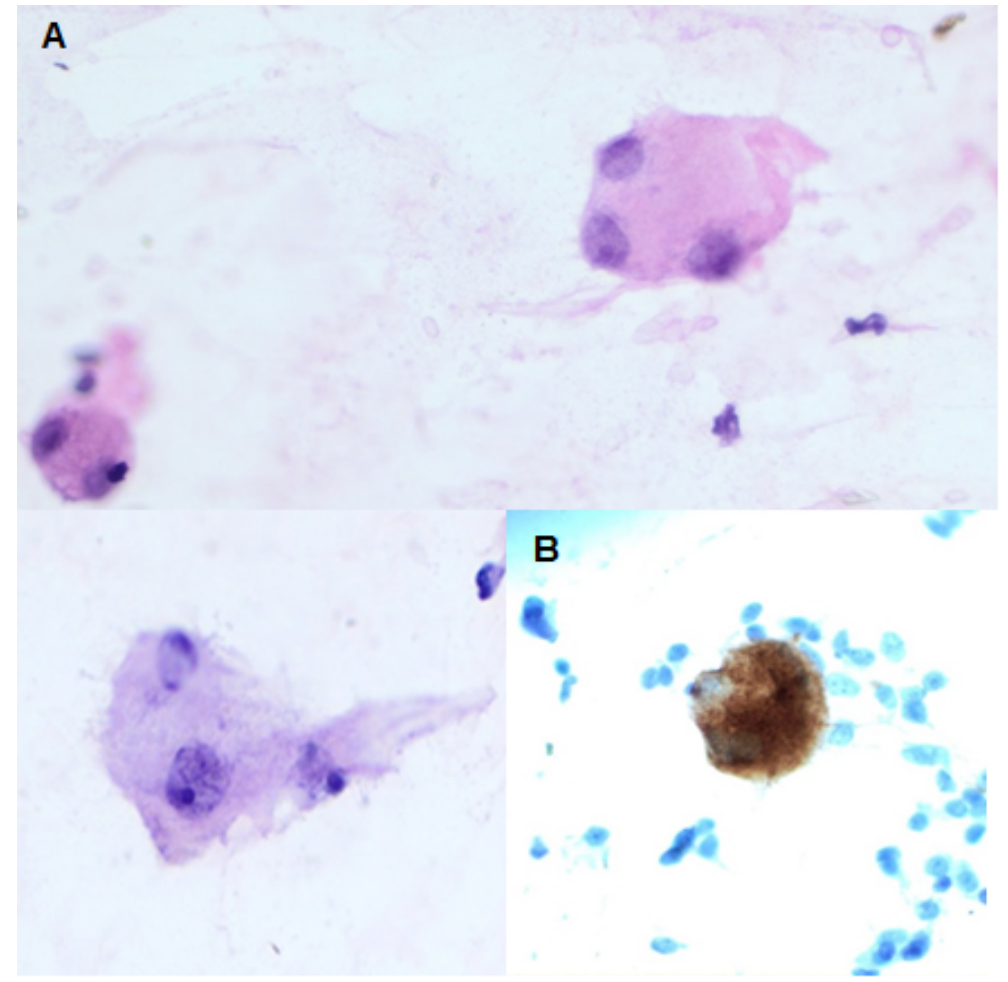

Figure 5: Ganglion cells in the FNA specimen - identified on Hematoxylin and Eosin stain (A) and immunohistochemical stain for neurofilament protein (B). (40X magnification).

DGP secreting somatostatin, serotonin, insulin, glucagon and/or pancreatic polypeptide have also been described [8]. On ultrasound DGP appears as an isoechoic mass, and on computed tomography as a homogenously hypoattenuated lesion located in the submucosa or muscularis propria of the duodenum [14]. Endoscopically it usually appears as a submucosal pedunculated lesion and, due to the submucosal location, preoperative endoscopic biopsies are usually inconclusive or initially diagnosed as ganglioneuromas or paraganglioma [1,14].

This lesion is usually centered in the submucosal layer of the duodenum; however, it can infiltrate the mucosa and/or the muscularis propria in more than $50 \%$ of cases [1]. DGP presents as a well circumscribed but unencapsulated submucosal nodule ranging in size from $0.5 \mathrm{~cm}$ to $10 \mathrm{~cm}$, with an average of $2.5 \mathrm{~cm}$ in largest diameter. Histologically DGP demonstrates a characteristic trilineage cellular population featuring epithelioid cells, spindle cells, and ganglion-like cells, each represented in varying proportions [15]. The spindle-shaped cells are usually randomly distributed or arranged in fascicles, demonstrate elongated nuclei with mild cytological atypia, and abundant eosinophilic cytoplasm. Immunohistochemically they are strongly positive for $\mathrm{S} 100$ and SOX-10; supportive of a peripheral nerve sheath differentiation [15]. The epithelioid cells are often arranged in nests separated by spindle cells and vascular septae, reminiscent of the Zellballen pattern of a paraganglioma, and are positive for synaptophysin and chromogranin; supportive of their neuroendocrine origin. Intermixed within these two cell types, a few scattered ganglion-like cells are present, expressing both neuro- endocrine and neuronal markers such as neuron-specific enolase (NSE), and NFP. Mitoses are usually rare, and no necrosis is generally identified [16]. The proportion of the three cell types varies widely, and depending on the predominant cellular component, DGP may mimic a Schwannoma, GIST or Leiomyoma, if the spindle-cell component predominates. If there is a predominance of epithelioid cells, the differential diagnosis may include a low-grade adenocarcinoma and well-differentiated neuroendocrine tumors. Rarely ganglion-like cells may predominate, and the diagnosis of a ganglioneuroma may be considered.

Description of the cytological appearance of DGP sampled by US-guided FNA are very few, and to the best of our knowledge, only two cases have previously been reported in the English language literature $[6,8]$.

In 2013, Dustin, et al. published the first cytology case report of DGP. The authors described a 56-yearold female with a $1.8 \mathrm{~cm}$ submucosal DGP in the second part of the duodenum. The lesion was transmurally penetrating the duodenal wall and demonstrated metastatic disease to retroperitoneal and peripancreatic lymph nodes. The aspirate was described as hypercellular and predominantly composed of epithelioid cells, with only a minor component of spindle cells and ganglion-like cells [6]. In the other case report, Lei, et al. described a 45 -year-old male with a $1.5 \mathrm{~cm}$ ampullary mass. This was a functionally active lesion with secretion of somatostatin and serotonin and demonstrated metastatic disease in regional lymph nodes. The duodenal aspirate was described as hypercellular, with a clean background 
(without tumor diathesis), again showing a dominant epithelioid component along with minor components of spindle and ganglion-like cells [8]. In our case the aspirate was normocellular and the predominant cellular component consisted of fascicles of spindle cells, with only a minor component of epithelioid cells and rare ganglion cells, which were identified only on retrospective review of the aspirate. No mitosis or necrosis was identified, and on follow-up, no metastatic disease has been identified to date.

In contrast to cases previously described in the literature, which were hypercellular with a predominant epithelioid cell component (in both primary tumor and lymph node) and presented with lymph node metastasis, the case we have described here was normocellular, predominantly comprised of clusters of spindle cells, and did not show evidence of nodal metastasis. Though no definitive association may be identified from this small sample size, a future larger study may further elucidate this observation to show if there is indeed a correlation between epithelioid predominant tumors and risk of lymph node metastasis.

The diagnosis of DGP can be more difficult on FNA if only a single cell lineage predominates. In such instances, a more broad differential diagnosis should be entertained. In fact, a predominant spindle cell component may mimic GIST, schwannoma or leiomyoma; while a predominance of epithelioid cells is suggestive of an adenocarcinoma, a low-grade neuroendocrine tumor (NET) or a paraganglioma. An abundant population of ganglion cells in a DGP could be misinterpreted as a ganglioneuroma [16]. In these cases, clinical information, lesion location, and appropriate use of immunohistochemical markers can often distinguish these neoplasms from each other.

\section{Conclusion}

In conclusion, in the evaluation of submucosal duodenal lesions by US-guided FNA, the possibility of a DGP should be included in the differential diagnosis when two or all three cell elements required for this diagnosis are identified and their phenotype is confirmed by immunohistochemical markers.

\section{Funding and Disclosures}

This manuscript was not funded, and the authors do not have any financial disclosures.

\section{Authors Contribution}

All authors have contributed equally at different stages of this manuscript.

\section{References}

1. Yoichiro Okubo, Megumi Wakayama, Tetsuo Nemoto, Kanako Kitahara, Haruo Nakayama, et al. (2011) Literature survey on epidemiology and pathology of gangliocytic paraganglioma. BMC Cancer 11: 187.

2. Barret M, Rahmi G, Duong van Huyen JP, Landi B, Cellier
C, et al. (2012) Duodenal gangliocytic paraganglioma with lymph node metastasis and an 8-year follow-up: a case report. Eur J Gastroenterol Hepatol 24: 90-94.

3. Rowsell C, Coburn N, Chetty R (2011) Gangliocytic Paraganglioma: a rare case with metastases of all 3 elements to the liver and lymph nodes. Ann Diagn Pathol 15: 467-471.

4. Kim JY, Hong SM (2016) Recent updates on neuroendocrine tumors from gastrointestinal and pancreatobiliary tracts. Arch Pathol Lab Med 140: 437-448.

5. Cathcart SJ, Sasson AR, Kozel JA, Oliveto JM, Ly QP (2017) Duodenal gangliocytic paraganglioma with lymph node metastases: A case report and comparative review of 31 cases. World J Clin Cases 5: 222-233.

6. Dustin SM, Atkins KA, Shami VM, Adams RB, Stelow EB (2011) The Cytological Diagnosis of Gangliocytic Paraganglioma: A case report. Diagn Cytopath 41: 650-653.

7. Hartford L, Sherazadishvili A, Leslie K (2017) Duodenal gangliocytic paraganglioma with lymph node metastasis: $A$ systematic case review. Int J Hepatobiliary Pancreat Dis 7: 53-61.

8. Lei L, Cobb C, Perez MN (2016) Functioning gangliocytic paraganglioma of the ampulla: Clinicopathological correlations and cytologic features. J Gastrointest Oncol 7: S107-S113.

9. Dahl EV, Waugh JM, Dahlin DC (1957) Gastrointestinal ganglioneuromas: Brief review with report of a duodenal ganglioneuroma. Am J Pathol 33: 953-965.

10. Kepes JJ, Zacharias DL (1971) Gangliocytic paragangliomas of the duodenum. A report of two cases with light and electron microscopic examination. Cancer 27: 61-67.

11. (2010) WHO Classification of Tumors of the Digestive System. Bosman FT, Carneiro F, Hruban RH, Theise ND, IARC Press, Lyon, France.

12. Dookhan DB, Miettinen M, Finkel G, Gibas Z (1993) Recurrent duodenal gangliocytic paraganglioma with lymph node metastases. Histopathology 22: 399-401.

13. Li B, Li Y, Tian XY, Luo BN, Li Z (2014) Malignant gangliocytic paraganglioma of the duodenum with distant metastasis and a lethal course. World J Gastrenterol 20: 1545415461.

14. Oh JY, Nam KJ, Choi JC, Cho JH, Yoon SK, et al. (2008) Benign submucosal lesions of the stomach and duodenum: Imaging characteristics with endoscopic and pathologic correlation. Eur J Radiol 67: 112-124.

15. Loftus TJ, Kresak JL, Gonzalo DH, Sarosi Jr GA, Behrns KE (2015) Duodenal gangliocytic paraganglioma: A case report and literature review. Int J Surg Case Rep 8: 5-8.

16. Odze RD, Goldblum JR (2015) Surgical pathology of the GI tract, liver, biliary tract and pancreas. ( $3^{\text {rd }}$ edn), Saunders Elsevier, Philadelphia, Pennsylvania. 